\title{
CME Properties and Fuzzy Classification
}

\author{
Y. H. Tang \\ Department of Astronomy, Nanjing University, Nanjing, China
}

Z. Z. Han

Department of Mathematics, Southeast University, Nanjing, China

\begin{abstract}
In this paper, the theory and method of fuzzy classification are applied to analyse CME properties, with the use of the data in preliminary report during 1979-1981, the theoretical computation for CME category is performed. According to the original data of average properties for 9 structural classes of CME, the standardized value is obtained by average value and standard deviation. The fuzzy similitude matrix and equivalent matrix are built by use of the correlation coefficient transformation and the method of similitude coefficient, then we can divide 9 different structures into different categories according to different threshold value. Halo was the most energetic, and Single Spike, Diffuse Fan CMEs were the least energetic.
\end{abstract}

\section{Introduction}

The study of coronal mass ejections (CMEs) category is very important, in special for the significant events, which play an important role to affect space and earth environment. It is widely accepted that the properties of CMEs depended strongly on their structure. Such morphological differences would seem to provide an important clue to understand the nature of CMEs. The fuzzy clustering analysis is a better method to analyse CME properties, since the classic statistical analysis can't criticize exactly every structure, in especial for describing the category, which some properties are very strong, but others are very weak.

\section{Basic Principle and Method}

How to give an objective and scientific classification of 9 structural CME is an important question for analyzing CME properties. A series of quantitative results of CME classification are obtainable with apply fuzzy clustering analysis.

The works using fuzzy clustering analysis method to CME properties research include three main steps as following:

1. Normalizing the sample data.

Set 


$$
a_{i j}^{\prime}=\frac{a_{i j}-\bar{a}_{j}}{\sigma_{j}}
$$

there

$$
\overline{a_{j}}=\frac{1}{n} \sum_{i=1}^{n} a_{i j} ; \quad \sigma_{j}=\sqrt{\frac{1}{n} \sum_{i=1}^{n}\left(a_{i j}-\overline{a_{j}}\right)^{2}}
$$

$a_{i j}$ is the original value of $\mathrm{j}$ average property of $\mathrm{i}$ structral CME.

Compressing normalized data in $[0,1]$,

$$
A_{i j}=\frac{a_{i j}^{\prime}-a_{j \min }^{\prime}}{a_{j \max }^{\prime}-a_{j \min }^{\prime}}
$$

where $a_{j \max }^{\prime}$ and $a_{j \min }^{\prime}$ indicate maximal and minimal value respectively in $a_{1 j}^{\prime}, a_{2 j}^{\prime} \cdots a_{n j}^{\prime}$.

2. Calculating the similar matrices to make use of coefficient method of correlation.

$$
r_{i j}=\frac{\sum_{k=1}^{m}\left(A_{i k}-\bar{A}_{i}\right)\left(A_{j k}-\bar{A}_{j}\right)}{\sqrt{\sum_{k=1}^{m}\left(A_{i k}-\bar{A}_{i}\right)^{2}} \times \sqrt{\sum_{k=1}^{m}\left(A_{j k}-\bar{A}_{j}\right)^{2}}}
$$

where

$$
\bar{A}_{i}=\frac{1}{m} \sum_{k=1}^{m} A_{i k} ; \quad \bar{A}_{j}=\frac{1}{m} \sum_{k=1}^{m} A_{j k}
$$

Then,we can obtain the similar matrix $\tilde{R}$

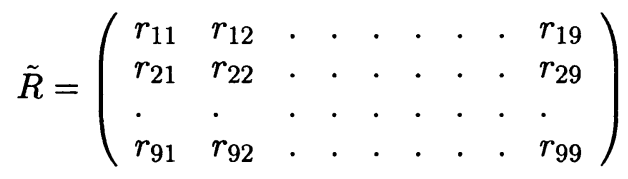

3.Madding Fuzzy equivalent matrices through the operational method of fuzzy relation as fllowing:

$$
\tilde{R}^{2}=\tilde{R} \cdot \tilde{R} ; \quad \tilde{R}^{4}=\tilde{R}^{2} \cdot \tilde{R}^{2} ; \quad \cdots \cdots ; \quad \tilde{R}^{2^{n}}=\tilde{R}^{2^{n-1}}=t(\tilde{R}) .
$$

$(\mathrm{n}=2,3, \cdots)$

Thus, the threshold value $\lambda(0 \leq \lambda \leq 1)$ will be determined as the clustering criterion according to clustering requirement,the clustering results can be determined. 


\section{Computational Results}

Based upon the fuzzy clustering analysis method as above, take the example of average properties of CME 1979 1981(Howard et al, 1985). Quantitative clustering results of 9 structural CME(Spike(1), Double Spike(2), Multiple Spike(3), Curved Front(4), Loop(5), Halo(6), Complex(7), Streamer Blowout(8) Diffuse Fan(9))had worked out and showed as table 1. Based upon the results showed as table 1, 9 structural CME can be classified into different categories according to different threshold values. For example, if we take the threshold value, $0.981<\lambda \leq 0.989,9$ different structures can be divided into 4 categories of CME importance of very strong(I), stronger(II), waeker(III), and very weak(IV).

Halo(6) was the most energetic, Complex(7), Curved Front(4), Double Spike(2), Multiple Spike(3), Loop(5) were stronger, Streamer Blowout(8) was weaker, and Single Spike(1), Diffuse Fan(9) were the least energetic.

Acknowledgments. This work was supported by the fund from NSFC.

\section{References}

Howard, R. A., Sheeley,J ${ }_{R}$ N. R., Koomen, M. J., \& Michels, D. J. 1985 JGR, 90,8173

Dubois, D., \& Prade, H.: 1980 Fuzzy Sets and Systems: Theory and Application, New York.

Table 1. Fuzzy equivalent matrices of nine strucyural CME

\begin{tabular}{llllllllll}
\hline & 1 & 2 & 3 & 4 & 5 & 6 & 7 & 8 & 9 \\
\hline$\tilde{R}^{4}$ & 1.000 & 0.989 & 0.989 & 0.986 & 0.989 & 0.474 & 0.989 & 0.291 & 0.992 \\
& 0.989 & 1.000 & 0.990 & 0.995 & 0.997 & 0.474 & 0.995 & 0.291 & 0.989 \\
& 0.989 & 0.990 & 1.000 & 0.990 & 0.990 & 0.474 & 0.990 & 0.291 & 0.989 \\
& 0.986 & 0.995 & 0.990 & 1.000 & 0.995 & 0.474 & 0.995 & 0.291 & 0.989 \\
& 0.989 & 0.997 & 0.990 & 0.995 & 1.000 & 0.474 & 0.995 & 0.291 & 0.989 \\
& 0.474 & 0.474 & 0.474 & 0.474 & 0.474 & 1.000 & 0.474 & 0.291 & 0.474 \\
& 0.989 & 0.995 & 0.990 & 0.995 & 0.995 & 0.474 & 1.000 & 0.291 & 0.989 \\
& 0.291 & 0.291 & 0.291 & 0.291 & 0.291 & 0.291 & 0.291 & 1.000 & 0.291 \\
& 0.992 & 0.989 & 0.989 & 0.989 & 0.989 & 0.474 & 0.989 & 0.291 & 1.000 \\
\hline$\tilde{R}^{8}=\tilde{R}^{16}$ & 1.000 & 0.989 & 0.989 & 0.989 & 0.989 & 0.474 & 0.989 & 0.291 & 0.992 \\
& 0.989 & 1.000 & 0.990 & 0.995 & 0.997 & 0.474 & 0.995 & 0.291 & 0.989 \\
& 0.989 & 0.990 & 1.000 & 0.990 & 0.990 & 0.474 & 0.990 & 0.291 & 0.989 \\
& 0.989 & 0.995 & 0.990 & 1.000 & 0.995 & 0.474 & 0.995 & 0.291 & 0.989 \\
& 0.989 & 0.997 & 0.990 & 0.995 & 1.000 & 0.474 & 0.995 & 0.291 & 0.989 \\
0.474 & 0.474 & 0.474 & 0.474 & 0.474 & 1.000 & 0.474 & 0.291 & 0.474 \\
0.989 & 0.995 & 0.990 & 0.995 & 0.995 & 0.474 & 1.000 & 0.291 & 0.989 \\
& 0.291 & 0.291 & 0.291 & 0.291 & 0.291 & 0.291 & 0.291 & 1.000 & 0.291 \\
0.992 & 0.989 & 0.989 & 0.989 & 0.989 & 0.474 & 0.989 & 0.291 & 1.000 \\
\hline
\end{tabular}

